\title{
Measuring Light Reflectance of BGO Crystal Surfaces
}

\author{
Martin Janecek, Member, IEEE, and William W. Moses, Fellow, IEEE
}

\begin{abstract}
A scintillating crystal's surface reflectance has to be well understood in order to accurately predict and optimize the crystal's light collection through Monte Carlo simulations. In this paper, we measure the inner surface reflectance properties for BGO. The measurements include BGO crystals with a mechanically polished surface, rough-cut surface, and chemically etched surface, and with various reflectors attached, both aircoupled and with coupling compound. The measurements are performed with a laser aimed at the center of a hemispherical shaped BGO crystal. The hemispherical shape eliminates any non-perpendicular angles for light entering and exiting the crystal. The reflected light is collected with an array of photodiodes. The laser can be set at an arbitrary angle, and the photodiode array is rotated to fully cover $2 \pi$ of solid angle. The current produced in the photodiodes is readout with a digital multimeter connected through a multiplexer. The two rows of photodiodes achieve 5-degree by 4-degree resolution, and the current measurement has a dynamic range of $10^{5}: 1$. The acquired data was not described by the commonly assumed linear combination of specular and diffuse (Lambertian) distributions, except for a very few surfaces. Surface roughness proved to be the most important parameter when choosing crystal setup. The reflector choice was of less importance and of almost no consequence for rough-cut surfaces. Pure specular reflection distribution for all incidence angles was measured for polished surfaces with VM2000 film, while the most Lambertian distribution for any surface finish was measured for titanium dioxide paint. The distributions acquired in this paper will be used to create more accurate Monte Carlo models for light reflection distribution within BGO crystals.
\end{abstract}

Index Terms-Lambertian reflection, light collection, Monte Carlo methods, scintillation crystal

\section{INTRODUCTION}

$\mathrm{T}^{\circ}$ accurately model a system with Monte Carlo simulation, each system component has to be accurately known and defined. Optical Monte Carlo simulations that model scintillating detectors require very accurate knowledge of the reflection off the scintillator surface, as small errors in

Manuscript received February 8, 2008. This work was supported by the National Nuclear Security Administration, Office of Defense Nuclear Nonproliferation, Office of Nuclear Nonproliferation Research and Engineering (NA-22) of the U.S. Department of Energy under Contract No. DE-AC02-05CH11231, grant number NNSA LB06-316-PD05 / NN2001000.

M. Janecek is with the Lawrence Berkeley National Laboratory, Berkeley, CA 94720 USA (phone: 510-486-5579; fax: 510-486-4768; e-mail: mjanecek@lbl.gov).

W. W. Moses is with the Lawrence Berkeley National Laboratory, Berkeley, CA 94720 USA (e-mail: wwmoses@lbl.gov). angular light distribution multiply through multiple reflections before the light is detected, and the error can grow quite large. However, the light reflection off crystal surfaces is poorly understood. Optical Monte Carlo software, such as DETECT [1], [2], Litrani [3], Geant4, [4], [5], or GATE [6-9], currently allow the operator to set the surface reflections as purely specular, purely diffuse (Lambertian), or a linear combination of specular and Lambertian, which might not be a true representation of the real world.

Our aim in this paper is to measure the reflectivity off BGO crystals for a variety of commonly used surface finishes, reflectors, and coupling methods. The results will be used to create more accurate simulation tools.

\section{BACKGROUND}

To model light collection, it is necessary to know the reflected light distribution from a beam of light that impinges on a surface at an arbitrary angle from inside of the scintillating crystal. Measuring this distribution using a rectangular BGO crystal is very difficult, if not impossible, as it is difficult to create a light beam incident at an arbitrary angle. In addition, collecting the reflected light from all angles is complicated by the refraction and total internal reflection at the escape surfaces of the crystal. Thus, to be able to measure the angular reflection distribution inside of a BGO crystal requires that we can 1) create a light beam inside of the BGO crystal that can be set to an arbitrary, well known incidence angle, and 2) measure all the reflected light off the surface over the entire $2 \pi$ of solid angle. The latter requirement suggests that refraction must be avoided as BGO has a very high refractive index $\left(n_{B G O}=2.1\right)$ and therefore can give rise to total internal reflections, making some angles impossible to measure. By using a hemispherical crystal, we can satisfy these requirements, as demonstrated in Fig. 1.

A laser is aimed towards the center of a $50.8-\mathrm{mm}$ diameter BGO crystal hemisphere. The light is reflected off the flat surface of the hemisphere and the light distribution is measured by a movable semi-circular array of photodiodes that can measure the entire $2 \pi$ of solid angle. The photo detectors are mounted at a constant radius to ensure equal solid angle coverage. All incident and reflected rays are perpendicular to the curved BGO crystal surface, thus eliminating any refraction and any total internal reflections. An instrument based on the principle above has been designed and built [10]. Some of the more important characteristics of 
this instrument are displayed in Table I, and a mechanical drawing is displayed in Fig. 2. The coordinate system used throughout this paper, defining theta and phi, is also displayed in Fig. 2. The instrument limits the size of the crystal to a maximum of $50.8 \mathrm{~mm}$ in diameter. Although a smaller size crystal is measurable, the larger crystal produces higher angular accuracy in the measurements [10]. The instrument was built for measuring many different types of crystals, and the laser wavelength was chosen accordingly. The laser wavelength, $440 \mathrm{~nm}$, lies close to the peak emission wavelength of BGO $(480 \mathrm{~nm})$, as well as other common scintillators such as $\mathrm{NaI}(\mathrm{Tl})(425 \mathrm{~nm})$, LSO $(420 \mathrm{~nm})$, and $\mathrm{LaBr}_{3}(380 \mathrm{~nm})$.

$\mathrm{BGO}$ is the first scintillator material we have measured with this instrument. The choice of BGO as the first crystal material to be examined was based on BGO's: 1) ability to be processed into a hemisphere, 2) non-hygroscopic properties, 3) common use and 4) relative affordable cost.

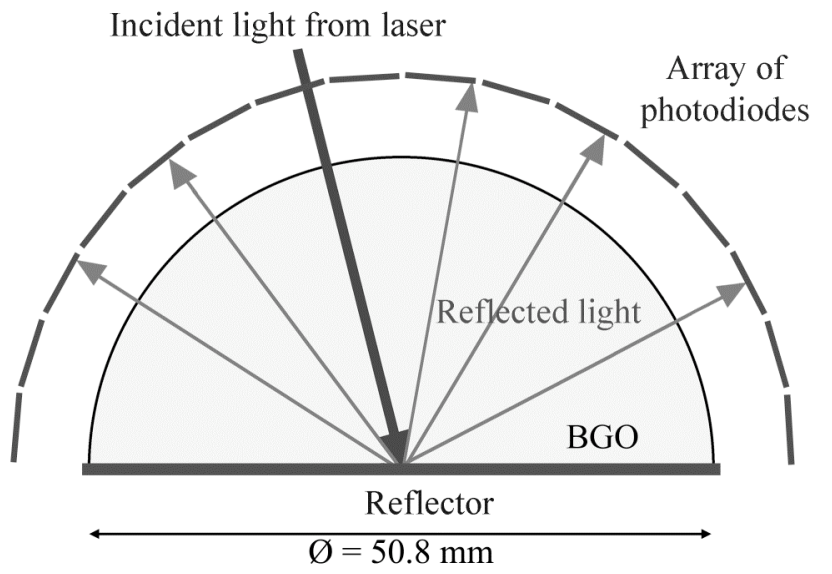

Fig. 1. Sketch (not to scale) of the BGO angular reflection measurement setup. Since all incident and reflected rays are normal to the surface, refraction effects are minimized and all angles of incidence and reflection can be measured.

The laser is a 440-nm, un-polarized, TEM $_{00}$, solid-state laser with $<0.8 \mathrm{~mm}$ beam diameter and $<1.0 \%$ power stability over 48 hours. The reflected light is measured by 36 Hamamatsu [Japan] S8729 PIN photodiodes. The $4 \times 5 \mathrm{~mm}^{2}$ photodiodes are mounted in two rows, offset to each other by half the length of a photodiode, with each photodiode subtending $6.3^{\circ}$ (in phi). Both the laser and the photodiode array can be positioned at any arbitrary angle - independently of each other - with 10 arc minute resolutions. The photodiode output currents are switched through a multiplexer to a digital multimeter, where the current is recorded. The current measurement gives us a dynamic range of $10^{5}: 1$. A LabVIEW program controls the motion of the laser and the photodiodes, the multiplexer switch, and the data collection. The mechanical set-up is housed inside of a light-tight box.

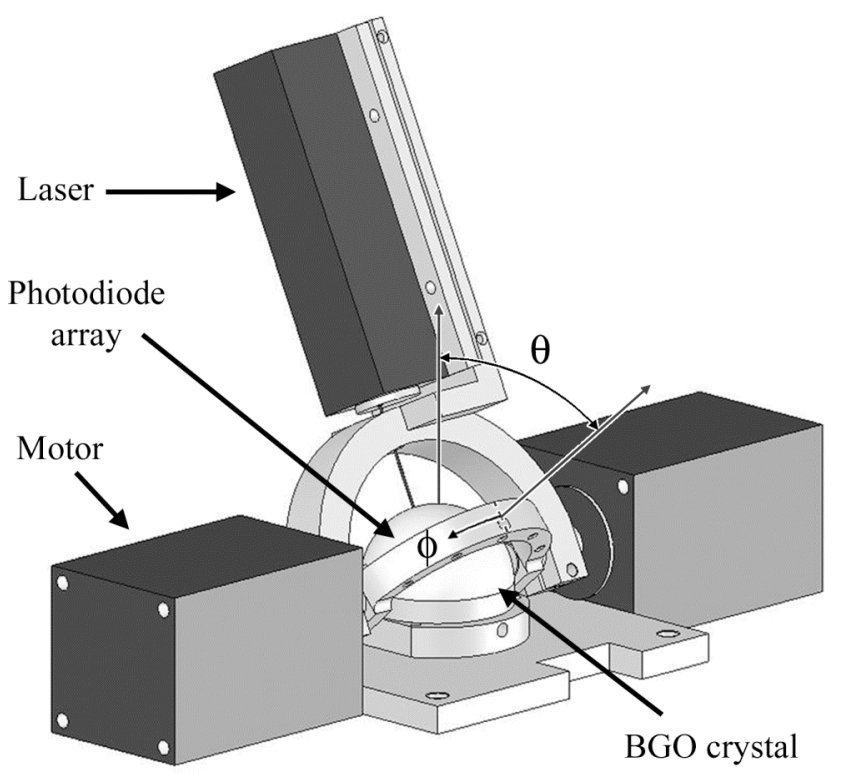

Fig. 2. Scale drawing of the instrument for measuring light distribution inside of BGO crystals. A laser, mounted on an arch, is aimed towards the center of a $\mathrm{BGO}$ hemisphere. The laser can, with a stepper motor, be rotated from theta $=$ $-90^{\circ}$ to $+90^{\circ}$. A photodiode array is mounted on a second arch, and can be rotated from theta $=-90^{\circ}$ to $+90^{\circ}$ with a second stepper motor. The theta and phi angles display the coordinates for the detectors, which are mounted on the inside of the detector arch. The entire set-up can fit inside of a $380 \times 370$ x $220 \mathrm{~mm}^{3}$ box (width $\mathrm{x}$ depth $\mathrm{x}$ height), with full rotational freedom for the laser.

TABLE I

INSTRUMENT CHARACTERISTICS

\begin{tabular}{lc}
\hline \hline Laser wavelength & $440 \mathrm{~nm}$ \\
\hline Laser power stability & $< \pm 1 \%$ over 48 hours \\
\hline Angular resolution & $<1^{\circ}$ \\
\hline Dynamic range & $10^{5}: 1$ \\
\hline Sampling & $4^{\circ}$ (theta) $\times 5^{\circ}(\mathrm{phi})$ \\
\hline
\end{tabular}

\section{MethodS}

Three BGO crystal cylinders [Saint-Gobain Crystals, Newbury, $\mathrm{OH}]$, each measuring $56.7 \mathrm{~mm}$ in diameter and $31.5 \mathrm{~mm}$ in height, were polished to $50.8-\mathrm{mm}$ diameter hemispheres [High Plains Optics, Inc., Longmont, CO]. All of the curved surfaces on the BGO crystals were polished to optical clarity. The flat surface was left in the original roughcut surface stage for one hemisphere, while one crystal was chemically etched (before polishing to the hemispherical shape), and the remaining crystal was mechanically polished. Various reflectors were attached to the irradiated flat surfaces, either by air coupling or by MeltMount ${ }^{\mathrm{TM}}$ [Cargille Laboratories, Cedar Grove, NJ] coupling $\left(n_{D}=1.582\right)$, as described in Table II. MeltMount ${ }^{\mathrm{TM}}$ might not be the most commonly used optical coupling material, but it is very similar to epoxy and other optical glues, in function as well as refractive index. MeltMount ${ }^{\mathrm{TM}}$ also has the advantage over glues that it is easily and completely removed from the crystal surface after an experiment so other reflector materials can be applied. The titanium dioxide paint was directly painted onto the surface. We also performed reflectance measurements on the BGO hemispheres without any reflectors attached. 
TABLE II

REFLECTORS USED

\begin{tabular}{|c|c|c|}
\hline Reflector & Coupling & Thickness \\
\hline Teflon $^{\mathbb{R}}$ [ACE Hardware, Oak Brook, IL] & Air & $230 \mu \mathrm{m}$ (3 layers) \\
\hline Lumirror $^{\mathbb{B}}[$ Toray, Japan $]$ & Air \& MeltMount ${ }^{\mathrm{TM}}$ & $240 \mu \mathrm{m}$ \\
\hline Tyvek $^{\circledR}\left[\right.$ DuPont ${ }^{\mathrm{TM}}$, Wilmington, DE] & Air & $150 \mu \mathrm{m}$ \\
\hline VM2000 [3M, St. Paul, MN] & Air \& MeltMount ${ }^{\mathrm{TM}}$ & $65 \mu \mathrm{m}$ \\
\hline Titanium dioxide $^{\dagger}$ & Painted on & $\sim 100 \mu \mathrm{m}$ \\
\hline None & - & - \\
\hline
\end{tabular}

Each BGO surface combination (surface treatment with a reflector applied) was examined for laser incidence angles from $+2^{\circ}$, in steps of $4^{\circ}$, to $+82^{\circ}$. For each laser incidence angle, the photodiode array was stepped from theta equal to $-90^{\circ}$, in $4^{\circ}$ steps, to $+90^{\circ}$. At each of these detector arch theta angles, all 36 photodiodes were read out. Each photodiode current measurement was averaged for $3 \mathrm{~s}$. (The multi-meter samples the current in discrete measurements, so this corresponds to 10 single measurements.) The acquired data was saved after each laser incidence angle into a text file for post-processing.

To account for photodiode dark current and any stray light, a background subtraction was performed for all the acquired data. The background was measured in a separate acquisition with a "black hole" in the center of the set-up. No BGO crystal was present at this measurement. The "black hole" was a hole cut through a black cloth with more black cloth covering the insides of the hole. The dark current background was measured to be less than $0.1 \mu \mathrm{A}$ for all photodiodes, while the corona from the laser produced a stray light peak less than $1 \mu \mathrm{A}$ for the (phi equal to) $0^{\circ}$ and $5^{\circ}$-photodiodes when the laser beam passed close to these detectors and illuminated a small portion of their (shielded) backsides. These background currents should be compared to $0.5 \mathrm{~mA}$ peak currents for a specular reflectance signal, and to $5 \mu \mathrm{A}$ peak currents for a diffuse reflectance signal.

We present our results on a $4^{\circ}$ (theta) $\times 5^{\circ}$ (phi) angular grid. The active area of a photodiode subtends $4^{\circ}$ in the thetadirection, and we therefore chose to step the photodiode array in $4^{\circ}$ increments, as mentioned earlier in this section. As for the phi-direction sampling, the photodiode centers are located $5^{\circ}$ apart in the two-row array. After calculating the precise location for each photodiode for each current measurement, our results were rebinned to this $4^{\circ}$ (theta) $\times 5^{\circ}$ (phi) angular grid using the assumption that the light intensity was uniform over the surface of each photodiode.

When the laser and detector arch are at the same theta angle, the detector arch blocks the laser beam. This creates some un-sampled areas for our angular distribution measurements. We have removed material from the supporting arch of the photodiode array to minimize the range of un-sampled angles and we perform linear interpolation for the remaining un-sampled areas. Signals that vary rapidly with angle, for instance specular reflection, cannot be accurately determined. However, slower varying functions, such as diffuse distributions, can easily be estimated with this linear interpolation.

Finally, the total reflectivity was calculated for each sample and each laser incidence angle by integrating the measured light over the full $2 \pi$ of solid angle. To get representative reflectivity values for the various combinations of surface treatments and attached reflectors, we calculated an average value for incidence angles from $14^{\circ}$ to $70^{\circ}$. We excluded the values for small incidence angles since we cannot accurately measure the specular reflections for these angles, and for very large incidence angles as these can contain larger $\left(>1^{\circ}\right)$ angular errors [10]. The reflectivity values were normalized to our previous measurements on four layers of $\mathrm{Teflon}^{\circledR}$ tape (310 $\mu \mathrm{m}$ thick) placed on a glass slide [11]. The absolute reflectivity for Teflon $^{\circledR}$ has been measured to be $99 \%$ by others [12-14].

\section{RESUlTS}

The reflectivity as a function of incidence angle for BGO crystals with no reflector attached is displayed for a mechanically polished surface, chemically etched surface, and rough-cut surface in Fig. 3. The reflectivity results for BGO crystals with reflectors attached are displayed in Table III.

For pure diffuse (Lambertian) reflectors, i.e., Teflon ${ }^{\circledR}$ tape [12-15] and titanium dioxide paint [11], attached to a mechanically polished surface, the Lambertian fraction and the specular fraction of the two light components were calculated and the results are displayed in Fig. 4. The Lambertian function was estimated by fitting the measured angular distribution to a cosine-function with the least square method. This function was then used to calculate the Lambertian portion of the signal. The remaining part of the signal was assumed to be specular.

Figs. 5 through 13 display the reflected light intensity at phi equal to $0^{\circ}$ for various combinations of surface treatments, reflectors, and coupling methods. Figs. 5, 6, and 7 display data for mechanically polished BGO crystal surfaces, Figs. 8, 9, and 10 display data for chemically etched BGO crystal surfaces, and Figs. 11, 12, and 13 display data for rough-cut BGO crystal surfaces, respectively. The data in Figs. 5, 8, and 11 were taken for laser incidence angle equal to $14^{\circ}$, the data in Figs. 6, 9, and 12 were taken for laser incidence angle equal to $34^{\circ}$, and the data in Figs. 7, 10, and 12 were taken for laser incidence angle equal to $62^{\circ}$, respectively. 


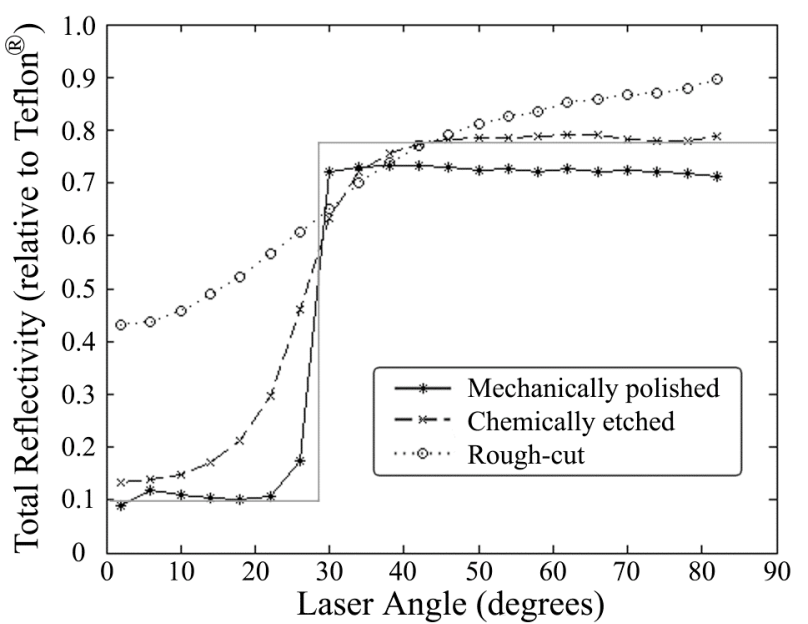

Fig. 3. Total reflectivity (over $2 \pi$ ) for a mechanically polished, chemically etched, and rough-cut BGO hemisphere without any reflector applied. The reflectivity has been normalized to four layers of Teflon ${ }^{\mathbb{B}}$ tape. The theoretical asymptotes (gray lines) for the mechanically polished hemisphere are $R_{\min }=0.096$, critical angle $=28.4^{\circ}$, and $\mathrm{R}_{\max }=0.776$.

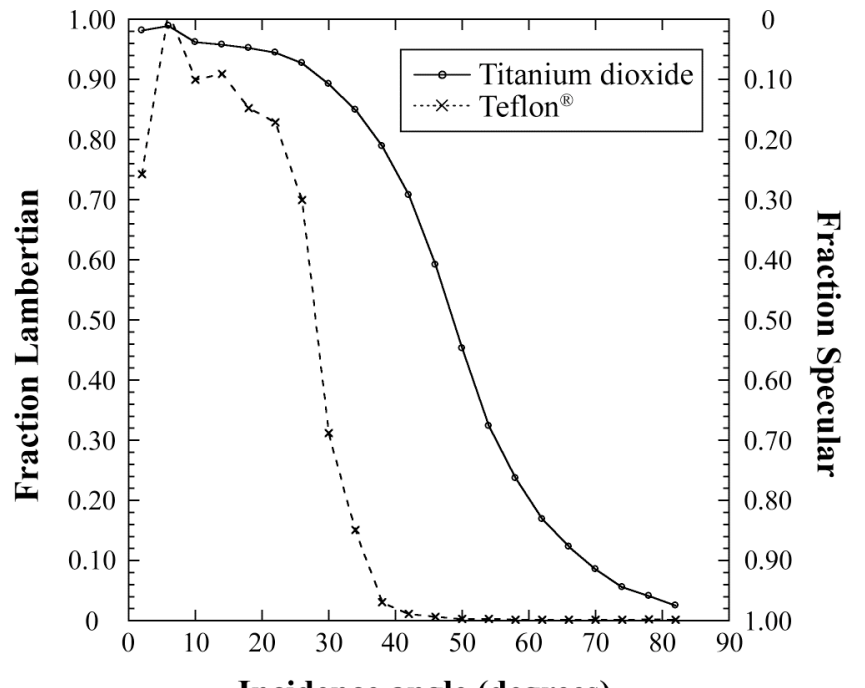

Incidence angle (degrees)

Fig. 4. Fraction Lambertian (left y-axis) and fraction specular light (right yaxis) for a mechanically polished surface with titanium dioxide paint and with Teflon ${ }^{\circledR}$ tape as a function of incidence angle. Notice that the Lambertian and specular fractions add up to 1.00 .

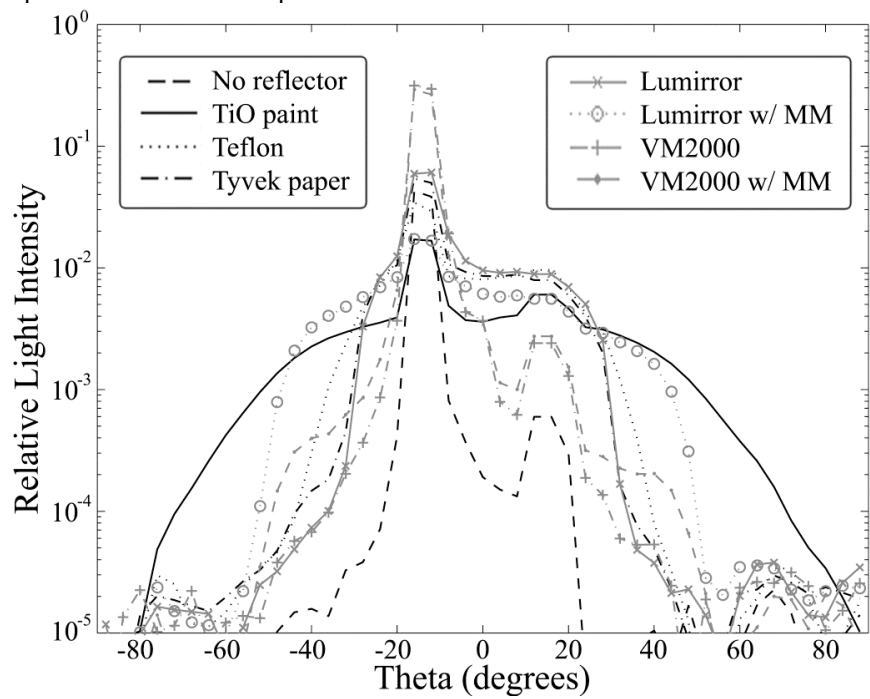

Fig. 5. Mechanically polished BGO crystal at theta incidence angle equal to $14^{\circ}$. The " $\mathrm{w} / \mathrm{MM}$ " indicates that the reflector is coupled to the scintillator with MeltMount ${ }^{\mathrm{TM}}$.

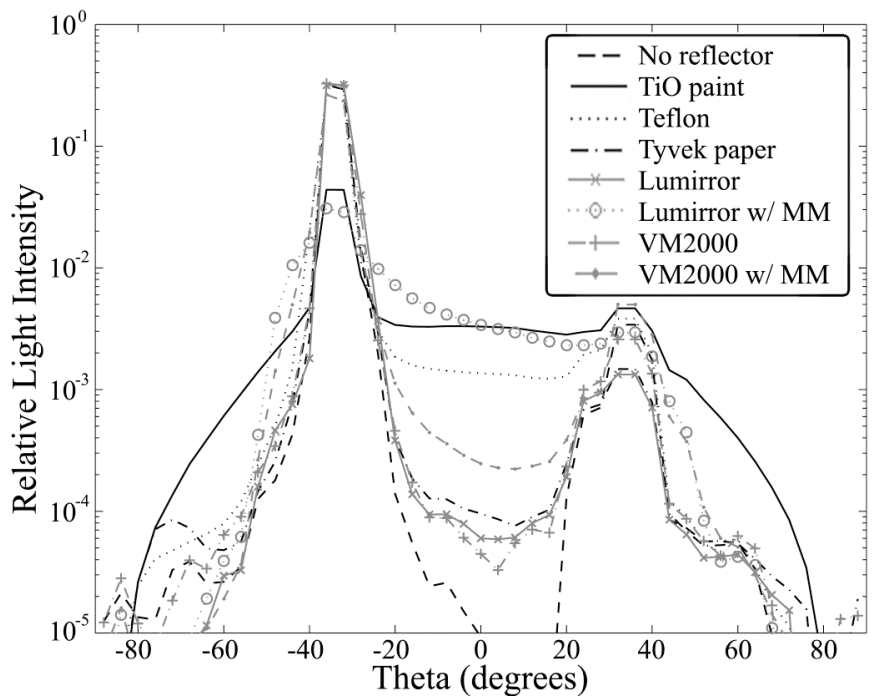

Fig. 6. Mechanically polished BGO crystal at theta incidence angle equal to $34^{\circ}$. The " $\mathrm{w} / \mathrm{MM}$ " indicates that the reflector is coupled to the scintillator with MeltMount ${ }^{\mathrm{TM}}$.

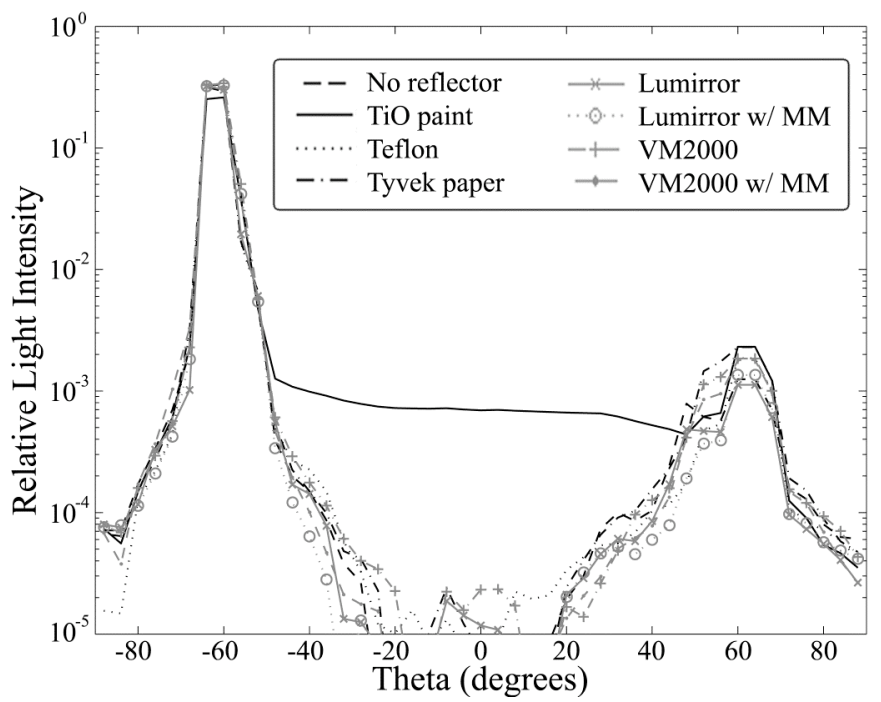

Fig. 7. Mechanically polished BGO crystal at theta incidence angle equal to $62^{\circ}$. The "w/ MM" indicates that the reflector is coupled to the scintillator with MeltMount ${ }^{\mathrm{TM}}$

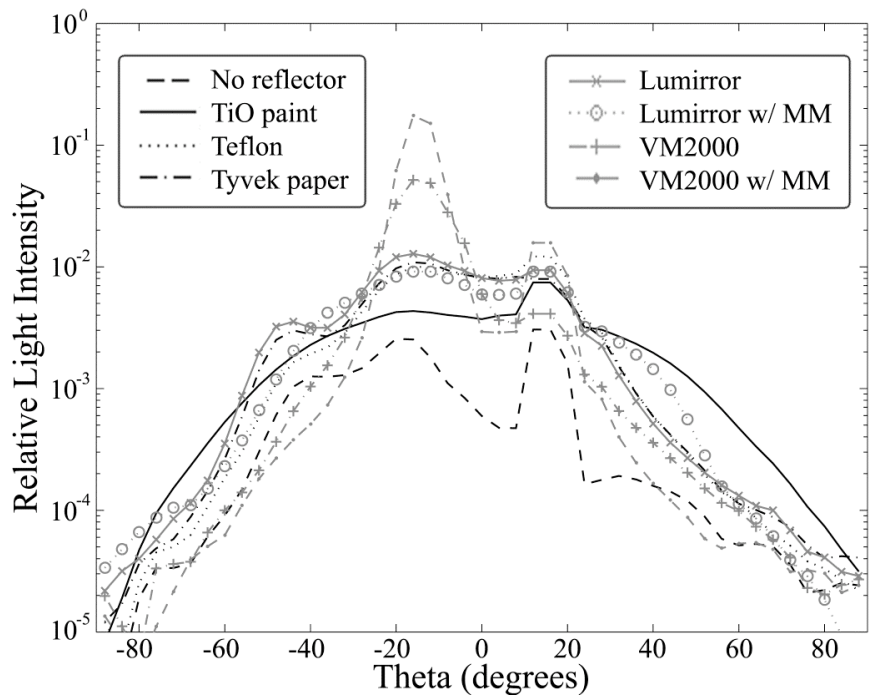

Fig. 8. Chemically etched BGO crystal at theta incidence angle equal to $14^{\circ}$. The " $\mathrm{w} / \mathrm{MM}$ " indicates that the reflector is coupled to the scintillator with MeltMount ${ }^{\mathrm{TM}}$. 


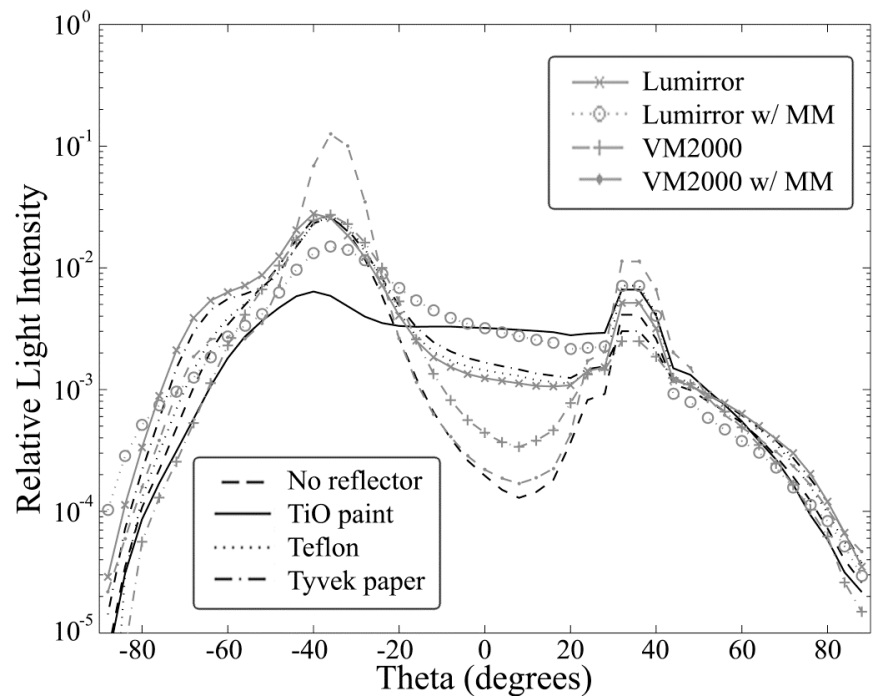

Fig. 9. Chemically etched BGO crystal at theta incidence angle equal to $34^{\circ}$. The "w/ MM" indicates that the reflector is coupled to the scintillator with MeltMount ${ }^{\mathrm{TM}}$.

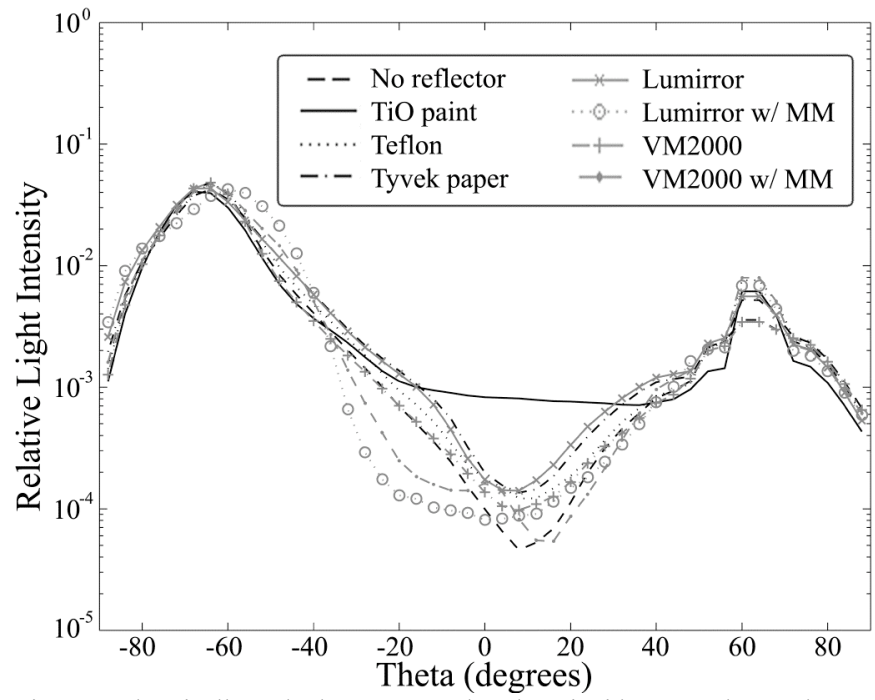

Fig. 10. Chemically etched BGO crystal at theta incidence angle equal to $62^{\circ}$. The " $\mathrm{w} / \mathrm{MM}$ " indicates that the reflector is coupled to the scintillator with MeltMount ${ }^{\mathrm{TM}}$.

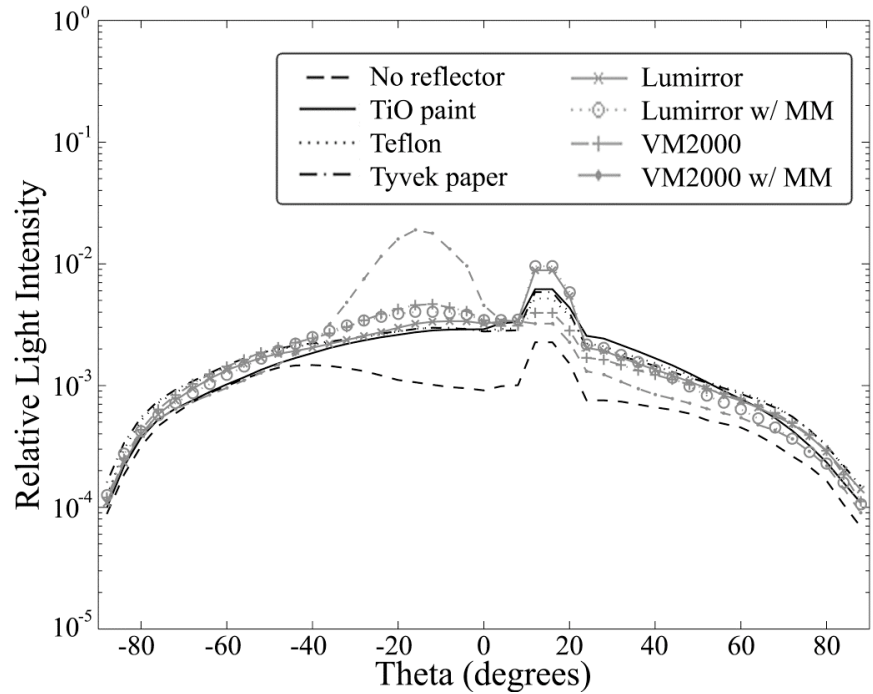

Fig. 11. Rough-cut BGO crystal at theta incidence angle equal to $14^{\circ}$. The " $\mathrm{w} /$ MM" indicates that the reflector is coupled to the scintillator with MeltMount ${ }^{\mathrm{TM}}$.

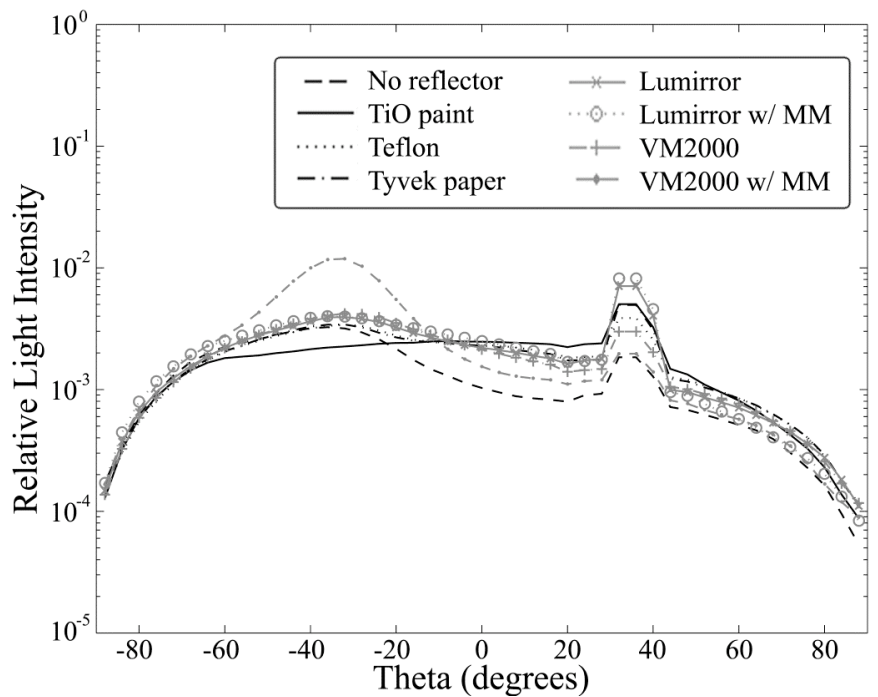

Fig. 12. Rough-cut BGO crystal at theta incidence angle equal to $34^{\circ}$. The "w/ MM" indicates that the reflector is coupled to the scintillator with MeltMount ${ }^{\mathrm{TM}}$.

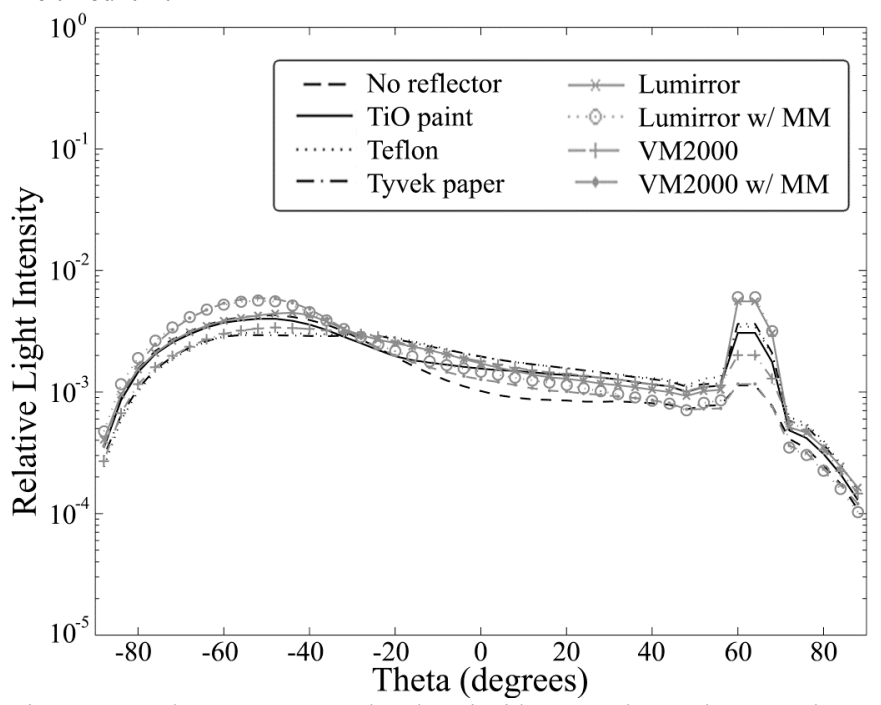

Fig. 13. Rough-cut BGO crystal at theta incidence angle equal to $62^{\circ}$. The "w/ MM" indicates that the reflector is coupled to the scintillator with MeltMount ${ }^{\mathrm{TM}}$.

TABLE III

TOTAL REFLECTIVITY (RELATIVE TO TEFLON ${ }^{\circledR}$ TAPE)

\begin{tabular}{lccc}
\hline Reflector & Rough-cut & Chem. etch. & Mech. pol. \\
\hline \hline Lumirror $^{\circledR}$ & $0.95 \pm 0.01$ & $0.80 \pm 0.02$ & $0.76 \pm 0.03$ \\
\hline Lumirror $^{\circledR}$ w/ MM & $0.93 \pm 0.01$ & $0.79 \pm 0.03$ & $0.79 \pm 0.01$ \\
\hline Teflon $^{\circledR}$ & $0.94 \pm 0.02$ & $0.78 \pm 0.01$ & $0.75 \pm 0.02$ \\
\hline Titanium dioxide $^{\circledR}$ & $0.89 \pm 0.03$ & $0.82 \pm 0.03$ & $0.81 \pm 0.01$ \\
\hline Tyvek $^{(B)}$ & $0.93 \pm 0.02$ & $0.78 \pm 0.02$ & $0.77 \pm 0.04$ \\
\hline VM2000 & $0.94 \pm 0.01$ & $0.78 \pm 0.01$ & $0.82 \pm 0.03$ \\
\hline VM2000 w/ MM & $0.89 \pm 0.02$ & $0.77 \pm 0.01$ & $0.77 \pm 0.03$ \\
\hline \hline Values are reported as averages for incidence angles $14^{\circ}$ to $70^{\circ}$ along with their standard deviations. The “w/ MM" indicates
\end{tabular}

that the reflector is coupled to the scintillator with MeltMount ${ }^{\mathrm{TM}}$.

\section{DisCUSSION}

The trends of the acquired data can be understood with simple optics laws. For a polished surface "coupled" to air (i.e., no reflector is attached), total internal reflection 
dominates above the critical angle. This is especially apparent in Fig. 3. Below the critical angle, we have a small specular reflection peak (caused by the Fresnel reflection at the BGOair interface), but most light escapes through the bottom of the BGO crystal surface without being detected. Below the critical angle, we should collect $9.6 \%$ of the incident light, as is calculated in (1), and above the critical angle (when we have total internal reflection at the reflection surface), we should collect $77.6 \%$ of the incident light, as is calculated in (2). These calculated values have been added to Fig. 3 as grey lines.

$$
\begin{aligned}
& (1-R) \cdot\left(R+R^{5}+R^{9}+\ldots\right) \cdot(1-R)=\frac{R \cdot(1-R)^{2}}{1-R^{4}} \approx 0.096 \\
& (1-R) \cdot\left(1+R^{2}+R^{4}+R^{6}+\ldots\right) \cdot(1-R)=\frac{1-R}{1+R} \approx 0.776 \\
& R=\left(\frac{n_{B G O}-n_{\text {air }}}{n_{B G O}+n_{\text {air }}}\right)^{2} \approx 0.126
\end{aligned}
$$

In the equations above, $R$ is the reflection coefficient, $n_{\text {air }}$ is the index of refraction for air, and $n_{B G O}$ is the index of refraction for BGO, respectively. Simple ray optics, where the light either gets reflected (R) or transmitted (1-R) when encountering a surface, was used to derive (1) and (2). The equations take into account all forward reflections, including second and higher order reflections, but disregards the back reflections (towards the laser). The Fresnel reflection [16], which is calculated in (3), is equal to $12.6 \%$ for BGO when setting the refractive index for BGO to 2.1. Applying Snell's law, the critical angle was calculated to be $28.4^{\circ}$.

The reason we do not collect $100 \%$ of the light above the critical angle is because the laser light is split between a backward reflection peak (from the air-BGO interface) and a forward reflection peak. This back reflection peak is visible for all the curves in Figs. 5 through 13 and is located at the incidence angle. We have in the equations above ignored the back reflection peak since we cannot measure it accurately (see the III. Methods section), and we therefore underestimate the light that will be collected. As can be seen in Fig. 3, the measured values agree fairly well with our analysis. The measured total reflectivity values are $8.9 \%$ higher than the calculated values below the critical angle (averaged for incidence angles of $2^{\circ}$ to $22^{\circ}$ ) and $6.7 \%$ lower above the critical angle (averaged for incidence angles of $34^{\circ}$ to $82^{\circ}$ ), respectively.

When we air-coupled a reflector to the BGO, the light distribution below the critical angle was dictated by the reflector's characteristic angular distribution, as can be seen in Fig. 5. Attaching Teflon $^{\circledR}$ tape produced a Lambertian distribution, however, there was also a specular reflection peak present, caused by the Fresnel reflection off the BGO bottom surface. The Lambertian and specular fractions of the light distribution were calculated and are displayed in Fig. 4 as a function of incidence angle; the Lambertian portion of the light is roughly $90 \%$ for incidence angles below the critical angle of $28^{\circ}$, and we have pure specular light reflections at high incidence angles. Air-coupling VM2000 film to the BGO produced pure specular reflections for all incidence angles, as can be seen in Figs. 5-7. Air-coupling Lumirror ${ }^{\circledR}$ or Tyvek $^{\circledR}$ produced in the same way angular distributions below the critical angle characteristic of the reflector materials [11], along with a specular peak.

Wetting the BGO surface with MeltMount ${ }^{\mathrm{TM}}$, or painting it with titanium dioxide paint, increased the critical angle. Attaching Lumirror ${ }^{\circledR}$ with MeltMount ${ }^{\mathrm{TM}}$ increased the critical angle to approximately $46^{\circ}$, compared to $28^{\circ}$ for air-coupled Lumirror $^{\circledR}$. There was no noticeable difference between aircoupled and MeltMount ${ }^{\mathrm{TM}}$-attached VM2000 film for a polished surface. This is an expected result as we have pure specular reflections for all incidence angles for both setups. Titanium dioxide paint, which is a Lambertian reflector [11], exhibited a critical angle of around $50^{\circ}$. The paint produced a linear combination of specular and Lambertian distributions, so we calculated the fraction of the two components and the results are shown in Fig. 4; the Lambertian distribution portion of the reflected light is above $90 \%$ for incidence angles below $30^{\circ}$, the specular component is as large as the Lambertian component at an incidence angle of about $50^{\circ}$, and the Lambertian component falls below $10 \%$ at incidence angles above $68^{\circ}$.

Chemically etching the surface widened the angular distribution compared to a polished surface; compare Figs. 810 with Figs. 5-7, respectively. This angular broadening of the angular distribution was even larger for roughened surface finishes, as can be seen in Figs. 11-13. On the microscopic level, a roughened surface consists of many quasi-randomly oriented small surfaces. The incident light beam gets exposed to many of these surfaces and their irregular orientations, which produces all three basic kinds of interactions (i.e., transmission, total internal reflection, and Fresnel reflection). For many photons, there will be multiple interactions. Therefore, high incident angles (that would have been above the critical angle for a polished surface) do not produce total internal reflection for all incident light, and low incidence angles (that would have been below the critical angle for a polished surface) produce total internal reflections for a fraction of the light, as can be seen in Fig. 3. Because of the surface irregularities, the roughened surface also produced diffuse reflections. With a roughened surface with an aircoupled reflector, the reflector material selection was of less importance, as can be seen in Figs. 11-13. The diffuse distribution for roughened surfaces can explain why the rough-cut surface produced the highest reflectivity values, as reported in Table III. When we have pure specular reflections, a significant portion of the light that is reflected off the BGO surface will eventually (because of Fresnel reflection) be reflected back towards the incidence angle. Since we underestimate the light intensity in this back reflection peak with our setup, we also underestimate the total light collected, and thus the reflectivity values. This effect is largest for polished surfaces and decreases with increasing surface roughness as the specular reflection peaks become wider in angle and therefore lower the central peak intensity. The measured reflectivity with our setup should therefore be the highest for rough-cut surfaces, followed by chemically etched, and lowest for the polished surfaces. 


\section{CONCLUSIONS}

In conclusion, we have accurately measured the reflection distribution off $\mathrm{BGO}$ surfaces inside of the crystal as a function of incidence angle and surface characteristics (surface treatment with various reflectors applied). Except for a very few surfaces, the data are not described by the commonly assumed linear combination of specular and diffuse (Lambertian) distributions. When selecting a BGO crystal setup with a surface finish, reflector, and coupling method, the surface roughness is the most important parameter. The reflector choice is of less importance and is almost of no consequence for rough-cut surfaces. The most Lambertian distribution for any surface finish was measured for titanium dioxide paint. The angular reflection distributions acquired in our measurements and presented in this paper will be used to create look-up-tables for optical simulation programs. This will aid in creating more accurate Monte Carlo models for light reflection distribution within BGO crystals.

\section{ACKNOWLEDGMENT}

This work was supported by the National Nuclear Security Administration, Office of Defense Nuclear Nonproliferation, Office of Nuclear Nonproliferation Research and Engineering (NA-22) of the U.S. Department of Energy under Contract No. DE-AC02-05CH11231, grant number NNSA LB06-316-PD05 / NN2001000.

\section{REFERENCES}

[1] G.F. Knoll, T.F. Knoll, and T.M. Henderson, "Light collection in scintillating detector composites for neutron detection," IEEE Trans. Nucl. Sci., pt. 1, vol. 35, pp. 872-875, Feb. 1988

[2] F. Cayouette, D. Laurendeau, and C. Moisan, "DETECT2000: An Improved Monte-Carlo Simulator for the Computer Aided Design of Photon Sensing Devices", Proc. SPIE Photonic North, Quebec, Canada, June 2002

[3] F.-X. Gentit, "Litrani: a general purpose Monte-Carlo program simulating light propagation in isotropic or anisotropic media", Nucl. Instr. and Meth. A, vol. 486, no. 1-2, pp. 35-39, June 2002

[4] J. Allison, et al., "Geant4 Developments and Applications", IEEE Trans. Nucl. Sci., vol. 53, no. 1, pp. 270-278, February 2006

[5] S. Agostinelli, et al., "Geant4 - A Simulation Toolkit", Nucl. Instr. and Meth. A, vol. 506, pp. 250-303, 2003

[6] G. Santin, D. Strul, D. Lazaro, et al., "GATE: A Geant4-Based Simulation Platform for PET and SPECT Integrating Movement and Time Management”, IEEE Trans. Nucl. Sci., vol. 50, no. 5, pp. 15161521 , Oct. 2003

[7] D. Strul, G. Santin, D. Lazaro, et al., "GATE (Geant4 Application for Tomographic Emission): a PET/SPECT general-purpose simulation platform”, Nucl. Phys. B (Proc. Suppl.), vol. 125, pp. 75-79, 2003

[8] K. Assie, V. Breton, I. Buvat, et al., "Monte Carlo simulation in PET and SPECT instrumentation using GATE", Nucl. Instr. and Meth. in Phys. Research A, vol. 527, pp. 180-189, 2004

[9] S. Jan, G. Santin, D. Strul, et al., "GATE: a simulation toolkit for PET and SPECT", Phys. Med. Biol., vol. 49, pp. 4543-4561, 2004

[10] M. Janecek and W.W. Moses, "Design of an Instrument to Measure Optical Reflectance of Scintillating Crystal Surfaces", IEEE Trans. Nucl. Sci., (accepted for publication, in press)

[11] M. Janecek and W.W. Moses, "Optical Reflectance Measurements for Commonly Used Reflectors", IEEE Trans. Nucl. Sci., (submitted to)

[12] Labsphere, Reflections Newsletter, pp. 1-4, Sept. 1998

[13] V.R. Weidner and J.J. Hsia, "Reflection Properties of Pressed Polytetrafluoroethylene Powder", Journal of Optical Society of America, vol. 71, no. 7, pp. 856-861, 1981
[14] B. Waldwick, C. Chase, and B. Chang, "Increased efficiency and performance in laser pump chambers through use of diffuse highly reflective materials", SPIE Proceedings, vol. 6663, 2007

[15] B. Courreges-Lacoste, J.G. Schaarsberg, R. Sprik, and S. Delwart, "Modeling of Spectralon diffusers for radiometric calibration in remote sensing", Opt. Eng., vol. 42, no. 12, pp. 3600-3607, December 2003

[16] E. Hecht, "Optics”, 2nd Ed, Addison Wesley, Reading, MA, USA, 1987 